\title{
Jesusgeschichten als phantastische Literatur *
}

\author{
Hans Zirker
}

„Jesus war ein superman - das meint mehr als die Hälfte aller Bewohner in der Bundesrepublik (...) War Jesus ein supennan?" So beginnt ein Religionsbuch sein Kapitel zu den Wundergeschichten. ${ }^{1}$ Damit stellt es wenn auch auf eine etwas modische Art die Frage nach dem Verhältnis von Glaube, Wirklichkeit und Phantastik ${ }^{2}$ - freilich nur als attraktiven Einstieg, der schnell abgetan ist. Doch es lohnt sich, der Sache weiter nachzugehen.

\section{Grundsätzliche Verständnisvoraussetzungen}

\subsection{Die erledigte historische Frage - die bleibende hermeneutische Unsicherheit}

Dass biblische Erzählungen von historischer Realität weit entfernt sein können, ist schon lange eine bare Selbstverständlichkeit. Die Reaktionen darauf sind allerdings vielfältig. Offensichtlich sind mit der historisch-kritischen Einsicht nicht auch schon die Verlegenheiten ausgeräumt.

Theologisch wie didaktisch ist die Scheu verständlich, Erzählungen wie etwa die vom Turmbau zu Babel, von Mose am brennenden Dombusch, von Jesu Totenerweckungen, vom Erscheinen des Menschensohns zum Weltgericht einfach als „erfunden“, „ausgedacht" o. ä. zu qualifizieren. Damit wird nämlich nur das freie Spiel der Einbildungskraft betont, doch nicht zugleich irgendwelche Rückbindung des so Erzählten an Erfahrungen. „Ausdenken“ lässt sich Beliebiges - als zuverlässige Rede verantworten dagegen nicht.

Selbstverständlich sind solche Geschichten nicht ohne Wirklichkeitsbezug, aber den hat schließlich auf irgendeine Art alle fiktionale Literatur; denn sie muss notwendigerweise auf die Bestände unserer Realität zurückgreifen. ${ }^{3}$ Auch in den phantastischsten Geschichten kommen Stücke unserer Welt vor; die bizarrsten Dinge werden noch aus Materialien und Formen aufgebaut, mit denen wir auch sonst zu tun haben; die ungewöhnlichsten Situationen und Ereignisse zeigen Strukturen, die uns vertraut sind. Wäre es anders, stünden wir schließlich vor einem unverständlichen Chaos und fänden uns als Leser nicht zurecht. Der bloße Verweis darauf, dass eine Erzählung, wenn auch erfunden, so doch unsere Wirklichkeit verarbeitet, reicht also offensichtlich nicht hin, um ihr eine besondere Geltung

\footnotetext{
* Erstveröffentlichung in: der evangelische erzieher 35, 1983, 228-243, für die Online Publikation geringfügig geändert.

${ }^{1}$ Schalom. Ein Arbeitsbuch für den Religionsunterricht im 3. und 4. Schuljahr von Erich Bochinger u. a., Frankfurt a. M. ${ }^{1} 1973,106$.

2 Der Begriff des Phantastischen wird hier zunächst umgangssprachlich benutzt und nur indirekt im Gebrauch erläutert. Für eine weitere Erörterung, die hier nicht durchgeführt werden kann, sei vor allem verwiesen auf Reimer Jehmlich, Phantastik - Science Fiction - Utopie. Begriffsgeschichte und Begriffsabgrenznng, in: Christian W. Thomson / Jens Malte Fischer (Hg.), Phantastik in Literatur und Kunst, Darmstadt 1980, 11-33; ders., Science Fiction, Darmstadt 1980; Dieter Penning, Die Ordnung der Unordnung. Eine Bilanz zur Theorie der Phantastik, ebd. 34-51; Tzvetan Todorov, Einführung in die phantastische Literatur, Frankfurt a. M. 1975.

${ }^{3}$ Vgl. Wolfgang Iser, Der Akt des Lesens, München 1976, etwa 101-114: Die Situationsbi1dung fiktionaler Texte" 114-143: Bezugsfeld und Selektion des Repertoires fiktionaler Texte.
} 
zu verschaffen. Auch Märchen, Sagen, Science Fiction, Utopien bleiben je in ihrer Art immer noch auf unsere Realität bezogen und von ihr abhängig.

\subsection{Realitätsmaßstäbe}

Entscheidend dafür, ob wir in dem erzählerischen Aufbau eines Textes noch „unsere Welt“ erkennen können oder nicht, sind sicher die jeweiligen Handlungsbedingungen. Dazu gehören besonders die das Handeln leitenden Erwartungen im Blick auf bestimmte Kausalzusammenhänge und kommunikative Beziehungen:

Wenn in Erzählungen Ereignisse eintreten, mit denen wir in unserem Alltag nicht rechnen; wenn man in ihnen Verhaltensweisen und Reaktionen findet, die uns befremdlich oder gar unverständlich bleiben; wenn Akteure auftreten, denen wir sonst nie begegnen, dann dürfte es uns schwerfallen, einen solchen Text noch für „realitätsgerecht“ ${ }^{4} z u$ halten. Seine Welt hat deutlich andere Modalitäten als die unsere, d.h. hier und dort ist jeweils anderes möglich und wahrscheinlich, notwendig und wirklich.

Darüber hinaus können uns Erzählungen auch noch dann befremdlich sein, wenn in ihnen andere Werte gelten als für uns: wenn man in ihnen etwas für gut hält, was wir verachten, und verwirft, was wir schätzen. Wir sagen vielleicht auch dann, dass diese Erzählungen nicht „unsere Welt“ erkennen lassen. Aber solche Diskrepanzen der Wertung sind uns auch im Spektrum der kulturellen Verschiedenheiten und aufgrund des geschichtlichen Wandels vertraut. Mit ihnen kommen wir deshalb noch einigermaßen leicht zurecht, obwohl auch sie zu den entscheidenden Handlungsbedingungen zählen.

Diese Kriterien gelten selbstverständlich auch für die biblischen Geschichten und für die Frage, wie es um ihren Realitätsbezug steht: Offensichtlich ist es weniger ausschlaggebend, ob etwas „wirklich so geschehen“ ist, wie es erzählt wird; ob die genannten Orte auch auf einer unserer Landkarten eingetragen sind; ob die Personen tatsächlich zu den genannten Zeiten gelebt haben; dies alles berührt nicht den Transfer in die Verhältnisse unseres Handelns. Aber andererseits liegt unter den ausgeführten Realitätskriterien für biblische Texte noch ein anderes Ergebnis auf der Hand: Wir rechnen unter unseren Bedingungen nicht damit, dass jemand dem Sturm gebietet und dieser sich daraufhin legt; dass jemand mit sieben Broten viertausend Menschen sättigen kann; dass wir vom Satan oder einem Engel angesprochen werden; dass Tote wieder aus dem Grab herausgerufen werden können usw. Was auch immer wir in unserer Welt tun oder lassen, wir sehen derartige Ereignisse nicht vor. Sie tragen zu deutlich die Züge des Phantastischen.

Die Frage, ob es für Gott aber nicht doch möglich sei, so etwas zu bewirken und ob sich nicht vielleicht doch einmal derartiges ereignet haben könnte, spielt dabei keine Rolle. Selbst wenn eine Brotvermehrung historisches Faktum gewesen wäre, könnten wir die bib-

\footnotetext{
${ }^{4}$ Vgl. die Unterscheidung von „realitätsgerecht“ und „faktizitätstreu“ bei Hans Glinz, Textanalyse und Verstehenstheorie 1, Frankfurt a. M. 1973, 106-146. Diese Unterscheidung trägt für unsere Einschätzung der biblischen Fiktionalität und Phantastik nichts ein; sie könnte höchstens das Problem mit anderen Worten fassen: denn wonach soll man ausmachen, was noch als „realitätsgerecht“, wenn auch nicht „faktizitätstreu“, gelten soll?
} 
lische Erzählung - an den Voraussetzungen gemessen, von denen wir in unserem sonstigen Handeln ausgehen - nicht als realitätsgerecht einschätzen. Unsere Beurteilung solcher Texte ist also von historisch-kritischen Einsichten weitgehend unabhängig. Dass wir sie als fiktive, gar phantastische Geschichten lesen, ergibt sich vielmehr aus unserem umfassenden kulturellen Überzeugungsbestand. ${ }^{5}$

\subsection{Die Rückführungen des Phantastischen auf vorgegebene Realität}

Wer Erzählungen, die unseren Wirklichkeitskriterien in entscheidenden Punkten nicht genügen, dennoch ernsthaft auf unsere Welt beziehen will, muss danach Ausschau halten, wo er die vermittelnde Grundlage finden kann. Für die Jesus-Geschichten bieten sich vor allem drei Ansätze an:

a. Vielfach wird der Wirklichkeitsbezug auf der Ebene der in den Erzählungen vorgestellten Werte gesucht, so etwa, wenn Wundererzählungen vor allem mit dem Blick auf den Gegensatz von vorgegebener Not und bewirktem Heil, also von Übel und Gutem, gelesen werden. Sie können dann als moralischer Appell oder auch als Trost angesichts unserer Verhältnisse erscheinen: Wo wir in unserer Welt schlechte Zustände finden, sollen oder dürfen wir jeweils gute anstreben - freilich nach den Möglichkeiten unserer Realität, nicht nach den Ausmaßen der fiktiven Geschichte. Diese sagt, wenn sie auf unsere Verhältnisse zurückgenommen wird, etwa nur noch: „Es kann nicht so bleiben, wenn wir versuchen, zu denken und zu handeln wie Jesus.“ 6 Selbstverständlich sind hier in dem Vergleich („wie Jesus“) die phantastischen Elemente getilgt. „Die Bedeutung der Wunder heute (...) lässt sich an den Taten ablesen, die aus dem Glauben an Jesus entspringen." 7 Die Brotvermehrungsgeschichte etwa gewinnt in dieser Sicht ihren guten Sinn dann, wenn sie auf ihre Weise in das Bewusstsein ruft, dass Christen Brot in überraschender Fülle austeilen und die Hungernden sättigen. (Das Schulbuch fügt deshalb an dieser Stelle eine entsprechende Photographie der caritativen Fürsorge bei. $^{8}$

b. Eine weitere Art, die Wundergeschichten mit der uns gegebenen Realität zu vermitteln und dabei die phantastischen Elemente zurückzunehmen, liegt in der existentialen In-

\footnotetext{
${ }^{5}$ Vgl. hierzu auch meine weiterreichenden hermeneutischen Studien: Lesarten von Gott und Welt, Düsseldorf 1979, bes. 94-101: Die Ausgrenzung des Fiktiven, 101-109: Literatur in der Bewährung (http://duepublico.uni-duisburg-essen.de/servlets/DocumentServlet?id=11684); Narrative« Geborgenheit in einer problematisierten Welt?, in: Katechetische Blätter 101, 1976, 731-735 (http://duepublico.uni-duisburg-essen. de/servlets/DocumentServlet?id=11877); Erzählungen und Überzeugungen im gemeinsamen Geschick. Ein Beitrag zur Hermeneutik »narrativer Theologie«, in: Antonius H. J. Gunneweg / Henning Schröer (Hg.), Standort und Bedeutung der Hermeneutik in der gegenwärtigen Theologie (Bonner Akademische Reden 61), Bonn 1986, 77-100 (http://duepublico.uni-duisburg-essen.de/servlets/DocumentServlet?id=11010); Neue Zuflucht beim Mythos? Eine kulturelle Entscheidung im Vorfeld der Bibeldidaktik (http://duepublico.uni-duisburg-essen. de/servlets/DocumentServlet?id=11067); Sprachanalytische Religionskritik und das Erzählen von Gott, in: RpB 10/1982, 148-160, und: W. Sanders / K. Wegenast (Hg.), Erzählen für Kinder - Erzählen von Gott, Stuttgart 1983, 33-43 (http://duepublico.uni-duisburg-essen.de/servlets/DocumentServlet?id=11587).

${ }^{6}$ Schalom (s.o. Anm. 1), 109. Bezeichnenderweise wird im Anschluss daran, also innerhalb des Kapitels zu den Wundererzählungen - die Geschichte von Onesimus - Paulus - Philemon besprochen.

${ }^{7}$ Orientierung Religion. Religionsbuch für das 5./6. Schuljahr von Ulrich Becker (u.a.), Frankfurt a. M. ${ }^{1} 1973$, 80.

${ }^{8}$ Ebd.
} 
terpretation vor. Sie verzichtet nach Rudolf Bultmann überhaupt darauf, das Verständnis der Erzählten Wunder auf äußere Ereignisse der Welt zu beziehen. „Vom Wunder reden heißt, von der eigenen Existenz reden, das heißt davon, dass in meinem Leben Gott sichtbar geworden ist" - wenn mich nämlich die "Offenbarung“, „das von Gott gesprochene Wort der Vergebung“, getroffen und verändert hat. ${ }^{9}$ In seinem neuen Selbstverständnis gewinnt der Gläubige zugleich auch ein neues Verhältnis zur Welt. Dieses wird in den Wundererzählungen ausgesprochen: Wer glaubt, begreift sich nicht mehr aus der ihm „verfügbaren Arbeitswelt“10, in der es auf seine Leistung ankommt, sondern aus der Schöpfung, in der er sich von den Taten Gottes abhängig sieht. Wenn in den Wundergeschichten die naturhaften Kausalzusammenhänge aufgehoben oder durchbrochen werden, ist dies nur ein Symbol dafür, dass der Gläubige seine eigene Existenz nicht nach den Ordnungen dieser Welt versteht. Bei einer solchen Interpretation treten an die Stelle der erzählten gewaltigen Ereignisse, dass Blinde sehen, Lahme gehen, Hungrige auf unerklärliche Weise gesättigt werden, Tote auferstehen usw. die Erzählerisch wenig tauglichen, aller Sinnenfälligkeit entkleideten Vokabeln „Offenbarung“, „Gnade“, „Wahrheit“. 11

c. Ein dritter Interaktionsweg sucht den Realitätsbezug der Wundergeschichten im Blick auf den Jesus zu gewinnen, der einerseits in den Texten durchgängig als zentraler Akteur vorkommt und andererseits ohne Zweifel eine historische Person ist, als solche für uns demnach offensichtlich nicht fiktiv. Die Überlieferungen, auch wenn sie schließlich von Phantastischem berichten, wollen von dem „wirklichen“ Jesus erzählen. Sie trotz ihres fiktiven Charakters an unsere Welt anzuschließen verlangt demnach anscheinend nur: wahrzunehmen, dass sie sich auf das irdische Leben Jesu zurückbeziehen und seine Bedeutung entfalten; dass sie damit also in den geschichtlichen Erfahrungen verankert sind, die die Menschen um Jesus mit inm gemacht haben. Das Phantastische hätte damit vor allem die Funktion, uns zur rechten Sicht seiner Würdestellung als von Gott Gesandter und Ermächtigter zu leiten. Es wäre nur „Ausschmückung“ im didaktischen Interesse des Glaubens.

Diese Sicht versöhnt anscheinend das Problembewusstsein der Entmythologisierung mit dem Bedürfnis nach geschichtlichem Realitätsbezug: Man weiß einerseits, dass Jesus nicht wirklich den Sturm mit seinen Worten zum Schweigen brachte, nicht wirklich Tote auferweckte usw., geht aber davon aus, dass die Erzählung doch den realen Jesus meint und ihn uns auf ihre Weise näherbringen will.

Es ist in dieser Sicht allerdings konsequent, wenn man versucht, nach Möglichkeit auf das Phantastische überhaupt zu verzichten und zu deutlicher „realitätsgerechten“ Texten zu kommen, nämlich durch einen uns weniger verstellten Bezug auf den historischen Jesus von Nazaret und sein Wirken in seiner Umwelt - über Texte, die den Maßstäben von

\footnotetext{
${ }^{9}$ Rudolf Bultmann, Zur Frage des Wunders, in: Ders., Glauben und Verstehen 1, Tübingen ${ }^{6} 1966,214-228$, hier 221 u. 228.

${ }^{10}$ Ebd. 222,

${ }^{11}$ Vgl. ders., Theologie des Neuen Testaments, Tübingen ${ }^{6} 1968,397$.
} 
„Wirklichkeit“ besser genügen. Wer fragt, ob Jesus ein „superman“ sei, richtet dann letztlich seinen Blick gar nicht mehr auf den „Helden“ der Wundergeschichte, sondern über ihn hinaus auf die vermeintlich reale Person, bei der sich diese Frage schnell erübrigt. Die Wundergeschichten sind bunte Illustrationen, die „deutlich zeigen, in welchem Sinne Jesus tatsächlich gehandelt hat“. ${ }^{12}$ Von dem außergewöhnlichen Wirken bleibt hier also nur noch die Intention bedenkenswert; alles übrige kann abgestreift werden, weil es nie faktisch gegeben war.

Alle drei genannten Interpretationsansätze lassen sich von den Wundergeschichten her rechtfertigen. Sie schließen einander auch nicht aus, sondern können einander ergänzen. Aber allen dreien ist gemeinsam, dass sie den Sinn der Erzählungen allein von dem her gewinnen wollen, was der gegebenen Realität und ihren Möglichkeiten entspricht - sei es in der geschichtlichen Vergangenheit Jesu, im Handlungsraum und -vermögen der heutigen Menschen oder in dem der Welt entzogenen Selbstverständnis der gläubigen Existenz. ${ }^{13}$ Demgegenüber bleibt zu überlegen, ob die phantastische Dimension der Jesusgeschichten nicht ihren eigenen Wert besitzt, so dass sie auch nicht als eine äußerliche Erzählweise abgestreift und durch andere Sprachformen gleichwertig ersetzt werden kann.

\subsection{Das Phantastische als Repräsentationsform des Glaubens}

In den Jesusgeschichten werden nicht nur irgendwelche neuen Erwartungen und Dimensionen eröffnet, sondern Bedingungen der alltäglichen Lebenswelt aufgehoben. Auch wenn dies biblisch nicht als eine „Durchbrechung von Naturgesetzen“ ausgelegt wird, weil man hier die Welt nicht als ein geschlossenes Kausalsystem sieht, so geschehen doch Dinge, die weit jenseits des üblichen Handlungsvermögens stehen. Jesus durchbricht die Grenze des menschlich Erwartbaren manchmal auf erschreckende Weise, so dass man ihn mit „großer Furcht" wahrnimmt (Mk 4,40), meint, „es sei ein Gespenst" und „aufschreit", sich „bestürzt“ zeigt und „außer sich gerät“ (Mk 6,49-51; auch 2,12; 5,42; Mt 12,23).

Einerseits betont man in religionspädagogischer Absicht immer wieder und vorrangig, dass Jesus kein „Zauberer“ gewesen sei; andererseits wird man - wenn man seinen Blick auf die Erzählenden richtet und die historische Frage zurückstellt - Jesus als einen Wundertäter sehen müssen, der über äußerst mirakulöse Kräfte verfügt und damit seine Umwelt in Staunen versetzt. Ohne Zweifel hat er mit Zauberern und Superman das gewaltige Handlungsvermögen gemeinsam. Gerade dies macht das didaktische Unbehagen gegenüber den sogenannten „Naturwundern“ verständlich. Doch diese phantastischen Ereignisse sind aus dem biblischen Jesusbild nicht zu eliminieren, sondern haben in ihm ihren festen Platz und ihre gute Berechtigung. Auf eine unersetzbare Weise belegen sie, wie sich der Blick des Glaubens nicht auf das Gegebene beschränken lässt, über die Bedingungen dieser Welt hinausgeht und sich auf neue Wirklichkeit einstellt.

\footnotetext{
12 Schalom (s.o. Anm. 1), 108.

$13 \mathrm{Vgl}$. auch die Kritik von Gerd Theißen an (religionsgeschichtlichen, redaktionsgeschichtlichen, traditionsgeschichtlichen und "historischen") Relativierungen und Entleerungen der Wundererzählungen in der Exegese (Urchristliche Wundergeschichten, Gütersloh 1974, 287-297).
} 
Der vorösterliche Jesus selbst griff zum Gleichnis, um auf diese Weise deutlich fiktiv und häufig auch mit bizarren Einzelzügen von der „Königsherrschaft Gottes“ zu sprechen. In dem Augenblick, in dem - nach Ostern - Jesus nicht mehr der Verkündigende war, sondern der Verkündigte wurde, musste er also auch selbst in Texte aufgenommen werden, die die Realitätsmaßstäbe unserer Handlungswelt überschritten. Dies geschah vor allem in der Auferstehungsverkündigung, die gar nicht anders als in phantastischer Sprache angelegt sein konnte. ${ }^{14}$ Die Überwindung des Todes ist kein empirisches Ereignis und nur mit dem Repertoire bildhafter Vorstellungskraft aussagbar. Hier können nicht vorliegende Sachverhalte wiedergegeben werden, weil es für „Auferstehung“ keine derartigen Sachverhalte gibt; aber nichts zu sagen hieße, die Macht des Todes zu belassen und der Hoffnung keinen Raum zu geben. So musste man erzählend Bilder entwerfen, die die verfügbare Realität weit hinter sich ließen, obwohl man nicht wissen konnte, wie das Neue, auf das sich der Glaube richtete, „an sich“ und „eigentlich“ ist. Wer nicht die Einbildungskraft zu Hilfe nimmt, kann nicht sinnvoll von „Gott”, „ewigem Leben“, „Erlösung“ usw. sprechen. Die Auferstehungsverkündigung ist unabdingbar auf die Phantasie des Glaubens angewiesen; man musste in ihr also Jesusgeschichten erfinden, wenn man überhaupt noch von ihm erzählen wollte. Ein „realer“ Jesus konnte nicht hinreichend Träger der christlichen Hoffnung sein, wie sie nach Ostern aufgebrochen ist.

Damit lag aber zugleich für die Überlieferungen vom vorösterlichen Jesus von Nazaret ein entsprechendes Erzählen nahe; denn Ostern sollte ja nicht schlechthin ein völlig neuer Anfang sein, sondern Rettung und Vollendung dessen, was vorausgegangen war. Wenn der vorösterliche Jesus mit dem nachösterlichen identisch sein sollte, musste demnach auch sein irdisches Leben als ein Teil der Geschichte erscheinen, die als ganze schließlich nur mit phantastischen Mitteln aussagbar ist. ${ }^{15}$

Auch wenn die Evangelien noch so viel historische Realität aus dem Leben Jesu verarbeiten, ihren Sinn als Glaubenszeugnis gewinnen sie gerade von den fiktionalen Partien her. Es ist die Paradoxie dieser Texte, dass sie mit den Mitteln der Phantasie die Härte der gegebenen Verhältnisse überwinden und die Zuversicht auf eine neue Wirklichkeit bekunden, die jetzt schon in der Gemeinschaft der Menschen, die einander lieben, beginnt und deren Grenzen nicht absehbar sind, so dass sie alle Vorstellungskraft übersteigt. Der Glaube muss in solchen Geschichten reden, weil er sonst nicht zeigen kann, wie seine Hoffnung über das menschlich Verfügbare und Machbare hinausgeht; und er kann dabei doch wissen, dass er seine Erzählungen erfunden und seine Bilder ausgedacht hat.

\footnotetext{
${ }^{14}$ Vgl. Alex Stock, Die matthäische Ostergeschichte, in: Ders./Manfred Wichelhaus, Ostern in Bildern, Reden, Riten, Geschichten und Gesängen, Zürich 1979, 179-200, hier 192ff mit Bezug auf Todorov (s. Anm. 2).

${ }^{15}$ Dass die Wundererzählungen die Funktion haben, den vorösterlichen Jesus als den auferstandenen Herrn erkennen zu lassen, betont unter den Religionsbüchern besonders: Wege des Glaubens. Unterrichtswerk für den Katholischen Religionsunterricht der Sekundarstufe 1, Bd. 2, Jahrgangsstufe 7/8, von Werner Trutwin und Klaus Breuning, Düsseldorf ${ }^{1} 1979,155$.
} 


\section{Die unterrichtliche Lektüre}

Im Unterricht zu lesen heißt immer auch zugleich, das Gelesene zu besprechen. Für biblische Texte gilt dies verstärkt, weil sie erstens vielfach schon zu bekannt sind, als dass die bloße Lektüre neue Einsichten verspräche, und bei ihnen zweitens erst ausgemacht werden muss, wie man sie überhaupt aufnehmen und welche Geltung man ihnen zuerkennen will. Im Folgenden werden die Gesichtspunkte entfaltet, die im Unterricht (etwa der Sekundarstufe 1) vor allem die Erarbeitung der Texte leiten könnten. (Selbstverständlich soll die dabei gewählte Systematik nicht auch die unterrichtliche Abfolge bestimmen; Fragen der methodischen Organisation sind hier nicht berücksichtigt.) Die beabsichtigte Lektüre ist nicht auf eine quasi-exegetische Detailarbeit ausgerichtet. Es soll im Gegenteil gerade um ein Lesen gehen, bei dem die Zuständigkeit weitgehend dem „normalen“ Leser zukommt und nicht dem Fachmann.

\subsection{Die Auswahl der phantastischen Texte}

Es mag vielleicht zunächst irritieren, wenn Jesus-Geschichten - entgegen üblicher religionspädagogischer Praxis - einmal gerade unter dem dominierenden Gesichtspunkt ihrer phantastischen Züge ausgewählt werden. Die Sichtung der gegenwärtigen evangelischen und katholischen Schulbuchreihen ergibt, dass man bei der Wunderthematik vorwiegend die Heilungserzählungen anspricht, mit denen man taktisch leichter zurechtkommt: Innen geht auf jeden Fall ein zeichenhaftes Handeln des historischen Jesus voraus, selbst wenn nicht zu entscheiden ist, ob dieses letztlich auf "natürlich zu erklärende Fähigkeiten“ zurückgeführt werden kann; eine solche Frage "liegt nicht im Blickpunkt der Bibel“. ${ }^{16}$ Dieses Aneignungsmuster ist aber bei den "Naturwundern“ nicht mehr brauchbar. So ist es erklärlich, dass von innen in den Schulbüchern fast nur noch die Brotvermehrung (Mk 6, 33-44; 8, 1-10 - offensichtlich wegen des naheliegenden symbolischen Bezugs zum Abendmahl und zur ethischen Verantwortung gegenüber den Hungernden) - und die Stillung des Seesturms (Mk 4,35-41 - wegen der Trostsymbolik angesichts menschlicher Gefährdungsängste) vorkommen. Um eine etwas größere Textgruppe zu erhalten, liegt es nahe, zu diesen beiden Erzählungen etwa die von Jesu Gang über den See (Mk 6,45-51), von den Totenerweckungen (Mk 5,21-24.35-43; Lk 7,11-17; Joh 11,1-45), von der Verklärung (Mk 9,2-10) und von Ostererscheinungen hinzuzunehmen. Wie auch immer die Textgruppe im einzelnen zusammengestellt sein mag (hier wurde z.B. das Weinwunder von Joh 2 wegen des besonderen theologischen Schwierigkeitsgrades ausgelassen), auf jeden Fall gehören vorösterliche und nachösterliche Geschichten zusammen. Anderenfalls wäre die theologische Bedeutung dieser fiktionalen Erzählweise nicht hinreichend verständlich zu machen.

Die Auswahl erfolgt von vornherein im Blick auf den phantastischen Charakter der Texte, ohne dass dieses Merkmal selbst schon genauer bestimmt wäre. Aber für den ersten Schritt reicht es hin, danach Ausschau zu halten, wo sich etwa die überlieferte Jesus-

\footnotetext{
${ }^{16}$ Ebd. 153.
} 
Geschichte am weitesten von unseren Realitätsmaßstäben entfernt. Selbstverständlich muss die Textgruppe nicht vom Lehrer vorgegeben sein, sondern kann auch von der Bibelkenntnis der Schüler her zusammengestellt werden. Dabei wird der Blick auch darauf gerichtet sein, in welcher Umgebung diese besonderen Erzählungen zuvor standen: nämlich inmitten anderer Erzählungen, die unserer Welt mehr entsprechen; die sich uns weniger massiv als „erfunden“ aufdrängen. Aber die Stücke, die wir herausheben, sind nicht einfach bizarres Sondergut, sondern Elemente, die unbefangen, ohne erzählerische Differenzierungen in den Gesamtbestand der Jesusüberlieferungen einbezogen wurden und damit deren Charakter entscheidend mitbestimmen.

\subsection{Die schlichte Erzählgestik}

Der Ungewöhnlichkeit der Ereignisse kontrastiert ihre einfache sprachliche Fassung. Wohl reagieren diejenigen, die die erstaunlichen Begebenheiten wahrnehmen, keineswegs gelassen; aber der Erzähler beschränkt sich durchweg darauf, ihre Bestürzung in knapper Form zu benennen; er führt deren „Entsetzen“ nicht vor (vgl. demgegenüber am ehesten noch Mt 28,4: „Die Wächter begannen vor Angst zu zittern und fielen wie tot zu Boden“); er lässt sich auch in seiner eigenen Erzählwelse nicht im geringsten von der Betroffenheit anstecken, sondern bleibt vielmehr völlig in sprachlicher Distanz und Nüchternheit; vor allem aber lässt er den „Helden“ selbst, der diese gewaltigen Taten vollbringt, ohne jedes pompöse oder theatralische Moment agieren: Jesus redet einfach zu („Mädchen, ich sage dir, steh auf!“, Mk 5,41f); er beschränkt sich auf wenige Handlungsschritte („Da nahm er die fünf Brote, blickte zum Himmel, sprach, brach ..., gab“, Mk 6,41); er vollzieht das sonst Unmögliche, als ob es das Gewöhnliche wäre (,In der vierten Nachtwache ging er auf dem See zu ihnen hin“, Mk 6,48); er hält den überwältigt Erstaunten die einfache Frage und die schlichte Aufforderung entgegen („Habt ihr etwas zu essen hier?“, Lk 24,41). Der schlichten Darstellungsweise entspricht es auch, dass alle diese Erzählungen in ihrem Umfang kurz sind; ihre Ereignisse sind schnell gesagt.

Der Vergleich mit sonstiger phantastischer Literatur, etwa Superman-Geschichten, führt einen gewaltigen Gegensatz vor Augen. Dort lebt die Phantastik entscheidend auch davon, wie sich ein Ereignis vollzieht und zur Sprache gebracht wird (die Sache ist ja vielfach recht banal). Hier dagegen liegt die erzählerische Pointe gerade darin, dass das Außergewöhnliche seine eigene Nüchternheit hat; dass das für unmöglich Gehaltene den Charakter des Naheliegenden und Selbstverständlichen annimmt. Damit erst wird mit besonderem Nachdruck angezeigt, dass andere Realitätsmaßstäbe Geltung gewinnen, neue Erwartungshorizonte sich eröffnen, die Orientierung in der Welt dieser Texte sich verändert.

\subsection{Die komponierte Realität}

Wenn die Erschließung der Texte nicht rein intuitiv und assoziationsgeleitet erfolgen soll, muss sie dem szenischen Aufbau der erzählten Situationen und Ereignisse nachspüren, z.B. den Grenzen und der Gliederung von Raum und Zeit, den Beziehungen der handeln- 
den Personen (oder auch anderer Akteure), den vorherrschenden Erwartungen, den geltenden Werten, den Ereignissequenzen und damit den Verschiebungen im gesamten erzählten Gefüge. ${ }^{17}$

\subsubsection{Die Einbettung der Ereignisse in überschaubare Raum- und Zeitverhältnisse}

Es sind keine ausladenden und weitreichenden Aktionen, von denen hier erzählt wird. Jesus ist unterwegs, wird angesprochen und geht in ein Haus (bei der Erweckung der Tochter des Jairus), er trifft am Stadttor einen Leichenzug (in Naim), sein Weg führt inn zu einem kurzen Aufenthalt auf einen Berg und wieder hinunter (bei der Verklärung), er fährt von einem Ufer des Sees zum anderen oder begegnet den Jüngern auf solcher Strecke (bei der Stillung des Sturms und dem Gang auf dem Wasser), er kommt in den Raum, in dem sich die Jünger versammelt haben (am Ostermorgen) usw. Es ist schon weit, wenn die Entfernung von jenseits des Jordans bis nach Betanien reicht (bei der Auferweckung des Lazarus) oder von Jerusalem nach Emmaus. Die Räume und Wege, die hier zur Sprache kommen, sind die der Alltagswelt. Hier werden nicht Himmel und Hölle oder andere phantastische Regionen einbezogen (allein die „Wolke“, aus der bei der Verklärung Jesu eine Stimme kommt, deutet in großer Zurückhaltung eine jenseitige Dimension an). Erst in der Folge eines solchen wunderbaren Ereignisses kann sich die räumliche Perspektive weit öffnen, wenn man nämlich von dem Geschehenen „überall in Judäa und im ganzen Gebiet ringsum“ (Lk 7,17) erzählt; aber auch damit bleibt die Erzählung deutlich in unserer Welt, in geographisch lokalisierbaren Gegenden.

Den überschaubaren Räumen und Wegen entsprechen die zeitlichen Ausmaße. Nur gelegentlich geht der Blick über die Grenzen eines Tages hinaus, etwa wenn wir lesen, dass es „bereits der vierte Tag“ nach dem Tod des Lazarus ist (Joh 11,39); dass die Menschen nichts mehr zu essen haben, weil sie sich „schon drei Tage“ bei Jesus in entlegener Gegend aufhalten (Mk 8,2); oder dass die Jünger enttäuscht aus Jerusalem weggehen, weil nach der Hinrichtung „heute schon der dritte Tag“ ist (Lk 24,21). Aber die zentralen Ereignisse selbst haben eine viel geringere zeitliche Erstreckung; sie wären eher in Stunden und Minuten zu fassen. Auch in dieser Hinsicht überschreiten die Geschichten also nicht die Maße, nach denen die Menschen alläglich ihre kleinen, bescheidenen Handlungen begreifen.

\subsection{2 Übliche und unübliche Akteure}

In den hier ausgewählten Geschichten kommt kaum jemand vor, den es nicht auch in ganz realistischen, den historischen Verhältnissen entsprechenden Texten geben könnte. Wir hören selbstverständlich von Jesus und den Jüngern (gelegentlich werden einige von ihnen ausdrücklich benannt, so vor allem zweimal Petrus, Jakobus und Johannes, Mk 5,37 und 9,2); wir hören von weiteren Freunden (wie Lazarus, Maria und Marta); dann einfach

\footnotetext{
${ }^{17}$ Zur einfachen und dennoch um Sorgfalt bemühten Strukturanalyse vgl. Hans Zirker u.a., Zugänge zu biblischen Texten, 2 Bde., Düsseldorf ${ }^{4} 2000 /{ }^{4} 1998$ (der Untertitel „Lesehilfe zur Bibel für die Grundschule“ betrifft nur die Textauswahl, nicht die Methode der Interpretation). - Die methodische Einleitung „Bibel-Lesen“ ist auch online verfügbar über http://duepublico.uni-duisburg-essen.de/servlets/DocumentServlet?id=11880.
} 
von „Leuten“, „vielen Menschen“, „5000 Männern“, „den Juden“; aus der Menge herausgenommen werden schließlich der Synagogenvorstand Jairus - mit Namen bezeichnet und dennoch sonst unbekannt - und eine Mutter, die um ihren Sohn trauert - anonyme Menschen und trotzdem in der Mitte des Geschehens. Diese Zusammenstellung der Akteure zeigt noch nichts Ungewöhnliches. (Anders wäre es für uns, wenn wir auch Erzählungen ausgewählt hätten, in denen Jesus Dämonen austreibt; aber damit wären wir gerade den historischen Gegebenheiten zur Zeit Jesu - den Vorstellungen und Erfahrungen seiner Zeitgenossen, an denen er teil hatte, und seinem wirklichen Handeln - besonders nahe.) Auch wenn Jesus dem Sturm gebietet und dieser gehorcht, durchbricht zwar das Geschehen die gewohnte Ordnung, doch die Naturmacht selbst gehört in den üblichen Erfahrungs- und Handlungsraum der Menschen.

Aber die Verhältnisse ändern sich entscheidend, sobald man zur Gruppe der Akteure auch (aus der Szene der Verklärung Jesu) Elija und Mose hinzunimmt. Wo sie „erscheinen“, wird der geschichtliche Abstand aufgehoben und die Grenze des Todes durchbrochen; in überraschender Schlichtheit stellt die Erzählung dabei fest: „sie redeten mit Jesus“ (Mk 9,4), als ob es sich um eine der vielen sonstigen Begegnungen handelte. Hinzu kommt in dieser Szene schließlich noch die "Stimme“, die aus der Wolke ertönt und Jesus als "geliebten Sohn“ proklamiert (V. 7). Damit erstreckt sich das Bezugsfeld der Akteure, so gewöhnlich es in diesen Geschichten sonst auch weithin besetzt ist, letztlich bis in die himmlische Welt.

Für alle ausgewählten Texte auffällig ist die Struktur der Beziehungen, das erzählte Soziogramm: immer ist ein und derselbe die zentrale Gestalt; im Verhältnis zu ihr gewinnen die anderen ihren Platz und haben teil am Geschehen - wenn sie sich Jesus zuwenden oder von ihm weggehen, ihn um Hilfe ersuchen oder sie abwehren, sich von ihm ansprechen lassen oder sich ihm verschließen, ihn verstehen oder ihn nicht begreifen. Dass sich gerade auch die jenseitigen Akteure ganz auf ihn richten, bekräftigt die herausragende und unvergleichliche Stellung, die diesen „Helden“ jeder sonstigen Position seiner Umgebung überlegen sein lässt.

\subsubsection{Die Realistik der Grenzsituationen}

Die überwältigenden Ereignisse der Wundergeschichten sind nur recht zu verstehen, wenn man wahrnimmt, worauf sie sich beziehen, d.h. bei der hier gegebenen Auswahl: auf Hunger, Bedrohung durch die Kräfte der Natur und Tod.

Eine Ausnahme scheint die Erzählung von der Verklärung Jesu darzustellen, da sie zunächst keinen äußeren Anlass, schon gar keine dringliche Not für dieses Ereignis erkennen lässt. Bei oberflächlichem Blick könnte dieses Wunder fast wie ein theologischer Luxus erscheinen: drei ausgewählten Jüngern wird ein exklusives Offenbarungserlebnts gegönnt. Aber bei einer solchen Einschätzung der Szene, würde man die Umgebung vernachlässigen, in die sie eingelassen ist: den Weg nach Jerusalem, die Ankündigung des Leidens, die Aufforderung zur Nachfolge in der Bereitschaft, sein Leben hinzugeben. Die Bitte des Petrus, auf dem Berg der Verklärung „drei Hütten zu bauen“ (Mk 9,5), geht aus 
dem Wunsch hervor, die harte und bedrohliche Realität der Gemeinschaft mit Jesus aufzuheben und statt ihrer die Ruhe himmlischer Wohnungen und die Beständigkeit ewiger Gemeinschaft zu gewinnen. Aber diese wird ihnen schließlich verweigert: Am Ende steht die irritierende Ernüchterung; die drei Jünger finden niemanden mehr vor außer Jesus, der mit ihnen den Berg hinabsteigt und seinen Weg weitergeht. Die himmlische Erscheinung ist letztlich im Kontrast bezogen auf die Kreuzigung.

Auch bei der Erzählung vom Gang Jesu über den See erscheint „das Motiv der Rettung stark zurückgedrängt“"18 und besonders die Epiphanie Jesu, also die Offenbarung seiner göttlichen Überlegenheit, betont. Dennoch sind die Elemente der Gefährdung unübersehbar: Der Einbruch der Nacht, bevor der Hafen erreicht ist, die vergebliche Mühe gegen den widrigen Wind anzukommen, die Anstrengung bis zum frühen Morgen (zur „vierten Nachtwache“ , Mk 6,48). Insgesamt lässt sich daraus doch das Bild einer Seenot gewinnen, auch wenn es erzählerisch nicht entfaltet ist (und den Verhältnissen des Sees Gennesaret nicht entspricht).

Die Wunder, so gewaltig sie auch angelegt sein mögen, beziehen sich nicht auf phantastische Bedürfnisse, sondern auf die Ängste der Gefährdung und der bedrohlichen Not. Sie sind Ereignisse in der Welt der kleinen Leute, in der sich die Erfahrungen der Schutz- und Ausweglosigkeit alltäglich aufdrängen. Das übermächtige Handeln Jesu steht gegen „die konkrete Negativität menschlichen Daseins“. ${ }^{19}$

\subsubsection{Die Durchbrechung der Erwartungen - Die Bekräftigung der Werte}

Damit wir mit unserer Welt und unserem Leben einigermaßen zurechtkommen können, wollen wir stabile Ordnungen, wollen wissen, woran wir - jetzt und in der Zukunft - sind, fürchten den Einbruch des Unvertrauten in unsere gewohnten Verhältnisse und Abläufe, reagieren erschreckt, wenn nicht das Übliche geschieht und unsere Erwartungen nicht mehr stimmen.

Diese Einstellung lassen nach den hier ausgewählten Wundergeschichten auch durchweg die Menschen um Jesus erkennen. Sie halten gerade nicht nach dem Wunder Ausschau, wie es schließlich geschieht: Sie wissen, was es heißt, tot zu sein, und lachen über den, der diesen Zustand nicht endgültig nehmen will (Mk 5,39f - als Jesus sagt, dass die verstorbene Tochter des Jairus nur schlafe); sie rechnen zwar mit der Auferstehung am "Letzten Tag" (Joh 11,24), doch keineswegs am heutigen unter den Bedingungen dieser Welt; sie wehren ab, wenn der Stein vom Grab weggerollt werden soll, da sie den Ablauf der Verwesung kennen (V. 39); sie gehen ratlos von Jerusalem weg, weil es „heute schon der dritte Tag" ist, seitdem durch die Kreuzigung Jesu ihre Hoffnung zerstört worden ist (Lk 24,21). Sie wissen, was es heißt, Hunger zu haben, und dass man in die umliegenden

\footnotetext{
${ }^{18}$ Rudolf Pesch, Das Markusevangehum, 1. Teil (= Herders theologischer Kommentar zum Neuen Testament 11/1), Freiburg 1976, 358.

${ }^{19}$ G. Theißen (s. Anm. 13), 285 und 295. Selbst zum Weinwunder von Kana betont Th., dass es „alles andere als ein ,Luxuswunder"“ sei. „Wo zum Feiern der Wein fehlt, dürften die Verhältnisse eingeschränkt sein. Gerade bei Feiern hat der kleine Mann Angst, durch seine Armut bloßgestellt zu werden." (250)
} 
Gehöfte und Dörfer gehen muss, wenn man sich „etwas zu essen kaufen“ will (Mk 6,36). Wer diese naheliegende Möglichkeit nicht ins Auge fasst, muss sich realistisch fragen lassen: „Woher soll man in dieser ungewohnten Gegend Brot bekommen, um sie alle satt zu machen?" (Mk 8,4). Sie wissen, welche Bedrohung ihnen auf dem See vom Sturm erwächst, und fragen deshalb denjenigen, der sich nicht darum zu sorgen scheint, vorwurfsvoll: „Meister, kümmert es dich nicht, dass wir zugrunde gehen?" (Mk 4,38)

Nirgends geht man in diesen Geschichten auf Jesus zu, um von ihm solche Taten zu erbitten. Offensichtlich hält man sie in den gegebenen Situationen nicht für möglich. Dies gilt freilich nicht für „Wunder“ überhaupt, im Gegenteil: Der Aussätzige kommt von sich aus zu Jesus und bittet um Hilfe (Mk 1,40-45); man bringt den Gelähmten, damit er geheilt werde (Mk 2,1-12); man kommt in der Zuversicht, dass Jesus den von einem Dämon Gequälten befreie (Mk 9,14-29) usw. Auch Jairus bittet Jesus, dass er seine Tochter, die im Sterben liegt, gesund mache (Mk 5,23), und Marta ist davon überzeugt, dass ihr Bruder Lazarus nicht gestorben wäre, wäre nur Jesus rechtzeitig gekommen, ihn zu retten (Joh 11,20). Dies all es ist aber noch im Bereich dessen, womit man in diesen besonderen Situationen zu rechnen vermag; doch in den Jesusgeschichten soll auch das noch geschehen, was selbst im Glauben nicht mehr für möglich gehalten wird. Gerade darin gewinnen diese Erzählungen ihre Phantastik, dass sie nämlich mit äußersten Nachdruck „auf die Durchbrechung menschlicher Erwartungen und alltäglicher Sinnwelt ${ }^{* 20}$ ausgerichtet sind. Jesus erscheint als derjenige, der Werke vollbringt, wie sie „kein anderer vollbracht hat“ (Joh 15, 24; vgl. auch 3,2; Mk 2,12; Mt 9,33). In dieser Sicht der Texte erweist sich Jesus durch sein Handeln auf einzigartige, unvergleichliche und unüberbietbare Weise als „Superman“.

Dem steht aber zugleich ein anderer Aspekt gegenüber, unter dem die Maßstäbe menschlichen Handelns nicht aufgehoben, sondern gerade kräftig bestätigt werden. Jesu Wunder bejahen Wertordnungen, nach denen sich die Menschen auch in ihrem Alltag ausrichten und von denen sie sich in ihren Wünschen und ihrem Tun leiten lassen: dass das Leben und die Gesundheit hohe Güter seien, Krankheit und Tod dagegen schlimme Übel; dass man sich mit Hunger und Angst nicht abfinden dürfe, sondern das grundlegende Bedürfnis aller anerkennen solle: in ungefährdeter Gemeinschaft zu leben und satt zu werden. ${ }^{21}$

Es wäre also unangebracht, sähe man in den Wundergeschichten nur die Aufhebung unserer Verhältnisse: So wie in der einen Hinsicht unsere Handlungsbedingungen durchbrochen werden, so kommen sie in der anderen voll zur Geltung. Phantastik und Realistik gehen in der Komposition dieser Texte eine spannungsvolle Einheit ein.

\footnotetext{
20 Ebd. 281.

${ }^{21}$ Selbstverständlich gibt es in diesen Erzählungen andererseits auch Werte, die zuvor nicht galten, z.B. mit Jesus in entlegener Gegend zu sein und auf seine Worte zu hören, statt in den Dörfern und Städten den üblichen Geschäften nachzugehen.
} 


\subsection{Die Aufarbeitung der phantastischen Geschichten durch heutige Leser}

„Mit den Wundern beginnt die Welt zum Reich Gottes zu werden“, heißt es in einem Schulbuch. ${ }^{22}$ Wie soll man dies aber auch noch von den machtvollen Ereignissen sagen können, die es nur als erzählte gibt, die also nur in Texten stehen? Diese Frage drängt sich unausweichlich auf, wenn man sich bewusst macht, wie in der biblischen JesusGeschichte „die historische Gestalt über alle Maßen hinaus gesteigert wird“. ${ }^{23}$ Den Verdacht, dass dies so sei, mögen viele Schüler ohnehin schon haben; der Religionsunterricht wird ihn bestätigen müssen. Dann aber bleibt ihm die entscheidende Aufgabe, ohne besondere theologische Komplikationen zu sagen, wie diese Texte auch nach solcher Einsicht noch ernst genommen werden können. Einfach und zugleich verantwortbar ist nur eine Möglichkeit: diese Wundererzählungen als Wunschgeschichten zu lesen. Sie sind „symbolische Handlungen“24, die sich mit der Welt, so wie man sie erfährt, nicht zufrieden geben - wie innerhalb dieser Geschichten auch Jesus symbolische Handlungen setzt: Brot austeilt, Kranke heilt usw. Freilich kann man dem Wunsch immer entgegenhalten, dass er die Verhältnisse noch nicht ändert; aber wenigstens „rebelliert er gegen die Realität und besteht darauf, dass sie sich in ihrer ganzen Handgreiflichkeit ändert, dass Kranke gesund, Hungernde satt, Blinde sehend werden“. ${ }^{25}$ Gerade die „Geschenkwunder" wird man „nur verstehen können, wenn man die in ihnen artikulierte Sehnsucht nach unendlich viel Brot, Fisch, Wein, kurz: nach Lebensmitteln für viele ernst nimmt“. ${ }^{26}$

Es fällt auf, dass die Religionsbücher bei ihrer Behandlung der Wunderthematik diesen Interpretationsweg nicht einschlagen (oder nur in schwachen Ansätzen nahelegen). Dabei wäre dies um so dringlicher, als sie häufig auf die Brotvermehrungsgeschichte eingehen, also gerade auf eines der „Geschenkwunder" von denen man sagen kann: keine andere Gattung „ist so sehr der Phantasie entsprungen wie diese“. ${ }^{27}$

Offensichtlich ist alles „Wunschdenken“ weithin so suspekt und für den Glauben gar durch Religionskritik als infantil und unverantwortlich diskriminiert, dass man es nicht wagt, diesen Charakter der Wundergeschichten deutlich aufzudecken. So bleibt den Schulbüchern nicht anderes übrig, als die besonders phantastischen Stücke der Jesus-Geschichte entweder beiseite zu lassen oder sie in ihren Gehalt kräftig zu reduzieren.

Wohl gibt es leichtfertige, gar zu naive und realitätsblinde Wünsche, aber andererseits auch solche, die die enttäuschende Wirklichkeit nicht ausblenden und sich gerade dabei als lebensnotwendig erweisen können. Die phantastischen Geschichten von Jesu Wirken übergehen die Härte unserer Wirklichkeiten nicht; das Wunderbare, das sie erzählen, ist immer nur punktuelles Ereignis, nur Episode. Wer davon hört, kann sich dabei gerade

\footnotetext{
22 Wege des Glaubens (s. Anm. 15), 157.

23 G. Theißen (s. Anm. 13), 279 (ähnlich 282).

24 Vgl. ebd. 229 die Überschrift zum 3. Teil.

25 Ebd. 286.

26 Ebd. 114.

27 Ebd. 113.
} 
„seiner ,normalen' Aussichtslosigkeit bewußt ${ }^{\text {}}{ }^{28}$ werden. Aber er vernimmt auch, dass hier auf eine bestimmte Weise - nämlich erzählend - der bestehenden Realität das Einverständnis verweigert wird.

Doch der Wirklichkeitsbezug der Wunschgeschichten beschränkt sich nicht auf diesen negativen Aspekt. Es kommen drei positive Momente des Erfahrungs- und Handlungsbezugs mit hinzu:

1. Wo gegen die Realität derart grundsätzlich und radikal Einspruch erhoben wird, besteht selbstverständlich die Verpflichtung, selbst etwas zu ändern, soweit man dies aus eigenen Kräften vermag. Es ist sicher notwendig, danach Ausschau zu halten, welche Forderungen in den Wundergeschichten stecken, auch wenn es falsch wäre, sie auf den Appell zu reduzieren.

2. Diese Geschichten stammen nicht aus der Beliebigkeit einer individuellen Phantasie, sondern sie sind aus den Erfahrungen mit Jesus von Nazaret hervorgegangen. In den Erzählungen wird sein reales Wirken und Geschick verarbeitet. Deshalb ist in dieser Hinsicht auch die Frage berechtigt, wie der historische Jesus bereits in einer überraschend neuen und machtvollen Weise handelte, so dass man inn schließlich in derart phantastische Erzählungen einbrachte.

3. Die Wundergeschichten weisen als symbolisches Handeln auf ein bestimmtes Selbstverständnis derer, die derartiges ernsthaft erzählen: sie nehmen die Grenzen ihres eigenen Vermögens wahr und zeigen, dass ihnen menschliche Daseinsbewältigung grundsätzlich nicht hinreicht.

Es bleibt bei alldem freilich das Unbehagen, dass die Jesus-Geschichten ursprünglich wohl nicht primär als Artikulationen des Wunsches, sondern vor allem als Überlieferung realer Begebenheiten verstanden worden sind. Man sah den symbolischen Bezug in den realen Ereignissen, während er sich heute weitgehend auf das Erzählen selbst verlagert hat. Dies betrifft die Gestalt unseres Glaubens fundamental, da er von seinem biblischen Ursprung her (auch wenn dies theologisch oft relativiert und verschleiert wird) wesentlich Wunderglaube ist. ${ }^{29}$ Wo man früher von einem Faktum erzählen hörte und aus ihm Hoffnung schöpfte, kann man heute oft nur noch den Wunsch vernehmen. Es liegt nahe, dass der Glaube damit auch nicht mehr gleichermaßen als Gewissheit erfahren wird, sondern eher als Bereitschaft, Entschluss und Versuch, die Welt und das Leben trotz des Dunkels und der Unstimmigkeit mit Zuversicht zu sehen. Vielleicht mag manchem, insbesondere von den Schülern, nicht gerade das Erzählen der biblischen Wunder heute noch als eine dazu geeignete symbolische Handlung erscheinen. Zunächst kommt es aber darauf an, diese phantastischen Geschichten überhaupt so zu verstehen.

\footnotetext{
${ }^{28}$ Ebd. 296.

29 Darauf besteht mit Nachdruck G. Theißen, ebd. insbesondere 287-297.
} 